\title{
Associação entre estado nutricional e qualidade de vida de idosos
}

\section{Association between nutritional status and quality of life in the elderly}

\author{
CAPPELARI, R. M. G. " ; LOCKMANN, A. S. 2,3; ZAFFARI, D. 1,3,4; \\ CAMPAGNOLO, P.D.B. ${ }^{1,4}$
}

\begin{abstract}
${ }^{1}$ Curso de Nutrição da Universidade do Vale do Rio dos Sinos - UNISINOS
2 Programa de Pós-Graduação em Ciências da Saúde da Universidade Federal de Ciências da Saúde de Porto Alegre - UFCSPA.

${ }^{3}$ Banco de Alimentos do Rio Grande do Sul.

4 Programa de Pós-Graduação Mestrado em Nutrição e Alimentos da Universidade do Vale do Rio dos Sinos - UNISINOS

* Autor correspondente- Denise Zaffari E-mail: Zaffari@unisinos.br
\end{abstract}

\section{DOI: https://doi.org/10.53817/1983-6929-ragg-v12n1-2}

\section{RESUMO}

Introdução: $O$ rápido envelhecimento da população e o aumento da longevidade traz desafios para os idosos, como a manutenção da saúde e da boa qualidade de vida. A má nutrição aumenta a morbimortalidade, reduz a funcionalidade e piora a qualidade de vida. Objetivo: Avaliar a associação entre o estado nutricional e a qualidade de vida de idosos atendidos no Projeto Passos da Longevidade do Banco de Alimentos do Rio Grande do Sul. Metodologia: Estudo transversal e retrospectivo com dados secundários, coletados entre março e dezembro de 2017, de um grupo de idosos não institucionalizados com 60 anos ou mais. As informações sociodemográficas foram obtidas por um questionário. Dados de peso e a altura foram coletados e o Índice de Massa Corporal (IMC) foi calculado. Os escores de qualidade de vida foram mensurados através do Questionário Short Form Health Survey 36 (SF-36). Resultados: 
Foram incluídos no estudo 185 idosos, com um IMC médio de $29,7 \mathrm{~kg} / \mathrm{m}^{2}$ sendo $41,1 \%$ deles obesos. A associação dos escores de qualidade de vida dos oito domínios do questionário SF-36, mostrou que os indivíduos obesos apresentaram pior escore nos domínios capacidade funcional e limitação por aspectos físicos, quando comparados aos idosos com baixo peso, eutrofia e sobrepeso ( $p<0,001$ e $p=0,042$, respectivamente). Conclusão: Os idosos obesos apresentaram pior capacidade funcional e limitação por aspectos físicos, quando comparados com aqueles com sobrepeso, eutrofia e baixo peso e maior limitação por aspectos físicos em relação aos idosos com sobrepeso.

Palavras-chave: Idoso. Qualidade de Vida. Estado Nutricional

\section{ABSTRACT}

Introduction: The rapid aging of the population and the increase in longevity brings challenges for the elderly, such as maintaining health and good quality of life. Poor nutrition increases morbidity and mortality, reduces functionality and worsens quality of life. Objective: To evaluate the association between the nutritional status and the quality of life of the elderly served in the Steps of Longevity Project of the Food Bank of Rio Grande do Sul. Method: Cross-sectional and retrospective study with secondary data, collected between March and December 2017, from a group of non-institutionalized elderly people aged 60 years or more. Sociodemographic information was obtained through a questionnaire. Weight and height data were collected, and the Body Mass Index (BMI) was calculated. Quality of life scores were measured through the Short Form Health Survey 36 (SF-36). Results: 185 elderly people were included in the study, with an average BMI of $29.7 \mathrm{~kg} / \mathrm{m}^{2}$ being $41.1 \%$ obese. The association of the quality of life scores of the eight domains of the SF-36 questionnaire, showed that obese individuals presented worse scores in the domains functional capacity and limitation by physical aspects, when compared to the elderly with low weight, eutrophy and overweight $(p<0.001$ and $p=0.042$, respectively). Conclusion: Obese elderly presented worse functional capacity and limitation by physical aspects when compared to those with overweight, eutrophy and low weight and greater limitation by physical aspects when compared to those with overweight.

Keywords: Aged. Quality of Life. Nutritional Status 


\section{INTRODUÇÃO}

O acelerado envelhecimento da população e o aumento da longevidade traz muitos desafios para os idosos, como a manutenção da saúde e uma boa qualidade de vida $(\mathrm{QV})$. Estudos epidemiológicos têm sinalizado que o risco de várias situações associadas ao envelhecimento, como problemas de saúde oral, diminuição da capacidade cognitiva e da autonomia, redução da percepção sensorial e da capacidade funcional e doenças crônicas, afetam a QV dos idosos. Essas situações podem ser amenizadas por meio de intervenções adequadas no estilo de vida, incluindo a adoção de uma alimentação saudável, a prática de atividade física, a cessação do tabagismo, a redução do estresse, entre outras. A má nutrição na idade avançada aumenta o risco de doenças, reduz a funcionalidade, piora a QV e aumenta a mortalidade (ANDRADE et al., 2017; PAULI et al., 2018 e KEHOE et al., 2018).

Vários são os fatores que levam o idoso a ter uma evolução nutricional desfavorável, como fatores fisiológicos, econômicos e biopsicossociais. Devido as alterações morfológicas, que são próprias do envelhecimento, há redução na eficiência da digestão, absorção e excreção de nutrientes que costumam ser frequentes, levando a atrofia da mucosa e diminuição da produção do ácido clorídrico. Também é comum a redução na produção da saliva e a perda da dentição devido ao uso de medicamentos ou por problemas na cavidade bucal (MAHAN et al., 2013). Além disso, a baixa condição econômica, a capacidade cognitiva e funcional prejudicadas, a depressão e o isolamento, juntamente com os demais fatores que influenciam o consumo alimentar e o estado nutricional dos idosos, contribuem para perda de peso não intencional, desenvolvimento de carências nutricionais ou excesso de peso que irá afetar, de maneira significativa, a saúde e a QV (SILVA e MURA, 2011; ANDRADE et al., 2017; HOLZ et al. 2013). Assim, a alimentação e o estado nutricional adequados representam condições essenciais do ser humano e se vinculam diretamente à saúde e a prevenção e reabilitação de agravos, sendo fundamentais para QV e o bem-estar da população idosa (ANDRADE et al., 2017; PAULI et al., 2018; KEHOE et al., 2018; FREITAS et al., 2017). 
O objetivo deste estudo foi avaliar a associação entre o estado nutricional e a QV da população idosa atendida pelo Projeto Passos da Longevidade do Banco de Alimentos do Rio Grande do Sul (RS). Este Projeto tem como objetivo a promoção e a manutenção da QV da população idosa que frequenta grupos de convivência nas instituições beneficiadas com a doação de alimentos pelo Banco de Alimentos, através de ações de educação em saúde. 


\section{MÉTODOS}

Este estudo é uma análise retrospectiva de um estudo transversal que utilizou dados secundários, coletados entre março e dezembro de 2017, do banco de dados do Projeto Passos da Longevidade, do Banco de Alimentos do RS. A população do estudo se caracterizou por uma amostra de conveniência, retirada do banco de dados de um estudo maior com 233 idosos. Foram incluídos no presente estudo idosos que, no estudo maior, responderam um questionário socioeconômico, o Short Form Health Survey (SF-36) e que tiveram suas medidas antropométricas aferidas. A coleta dos dados ocorreu nas Instituições onde os idosos participam de Grupos de Convivência, semanalmente, e foi realizada pela equipe de nutricionistas e acadêmicos de nutrição do Banco de Alimentos do RS.

A amostra do estudo foi composta por dados de 185 idosos não institucionalizados que responderam ao questionário socioeconômico e o SF-36 para mensurar a QV e que tiveram suas medidas antropométricas aferidas. Foram excluídos do estudo dados dos participantes dos grupos de convivência com idade inferior a 60 anos.

As informações coletadas do banco de dados, relacionadas aos aspectos sociais e econômicos incluíram a idade, o sexo, o nível de escolaridade, a cor da pele e a situação conjugal que foram compilados de um questionário com perguntas simples e diretas.

Os dados antropométricos extraídos do banco de dados foram: peso, estatura e Índice de Massa Corporal (IMC). O peso foi obtido pela aferição em balança eletrônica portátil da marca Plena, com capacidade para $150 \mathrm{~kg}$ e os indivíduos, quando foram pesados, estavam descalços, utilizando roupas leves e foram posicionados com os pés juntos e os braços estendidos ao longo do corpo. A estatura foi mensurada utilizando estadiômetro da marca Personal Caprice Portátil Sanny. Os participantes estavam descalços, com pés juntos e alinhados com a nuca, as nádegas e os calcanhares encostados na parede, sem adornos nos cabelos, olhando para um ponto fixo na altura dos olhos e com os braços estendidos ao longo do corpo. O IMC foi obtido pela divisão da massa corporal em quilogramas pelo quadrado da altura em metros - $\mathrm{kg} / \mathrm{m}^{2}$. Para classificar o IMC foram adotados os pontos de corte da Organização Pan- 
Americana de Saúde (OPAS): Baixo peso para IMC $<23 \mathrm{Kg} / \mathrm{m}^{2}$; Eutrófico para IMC de 23 a $28 \mathrm{Kg} / \mathrm{m}^{2}$; Sobrepeso para IMC de 28 a $30 \mathrm{Kg} / \mathrm{m}^{2}$ e Obesidade para IMC $>30 \mathrm{Kg} / \mathrm{m}^{2}$ (OPAS, 2003).

As aferições do peso e estatura foram realizadas por nutricionistas e acadêmicos de Nutrição do Banco de Alimentos do RS.

Os escores relacionados à QV foram mensurados através da aplicação do questionário SF-36, cuja confiabilidade e validade de constructo foram testadas com 1.980 pacientes britânicos, com idades entre 16 e 74 anos. É organizado em 36 itens, compostos por 8 domínios com várias questões. Após a avaliação, os escores foram codificados, somados e transformados em uma escala de 0 a 100, na qual quanto maior for a pontuação, melhor a QV (TAFT C et al, 2001).

Os dados coletados foram analisados com auxílio do programa estatístico SPSS - versão 21.0.

As variáveis quantitativas apresentaram normalidade pelo Teste de Kolmogorov-Smirnov e foram descritas por média e desvio padrão e as categóricas por frequências absolutas e relativas. Para comparar as médias dos escores de QV, a Análise de Variância (ANOVA) complementada pelo teste de Student Newman-Keuls foi aplicada. O nível de significância adotado foi de 5\% $(p<0,05)$.

Este estudo está de acordo com a Resolução o 466/2012 e a Resolução no 510/2016 e foi aprovado pelo Comitê de Ética e Pesquisa da Universidade do Vale do Rio dos Sinos - UNISINOS sob o número 3.510.865.

\section{RESULTADOS}

O banco de dados incluiu um total de 233 indivíduos. Destes, 48 foram excluídos (25 deixaram de comparecer aos encontros dos grupos; 19 tinham menos de 60 anos; 3 foram a óbito e 1 não apresentava os dados relacionados aos escores de QV), sendo que a amostra do estudo ficou em 185 indivíduos.

Embora a média do IMC tenha sido $29,7 \mathrm{~kg} / \mathrm{m}^{2}$, quando os indivíduos foram estratificados por faixa de IMC, foi observado um maior número de indivíduos com obesidade (IMC $\geq 30 \mathrm{~kg} / \mathrm{m}^{2}, \mathrm{n}=76 ; 41,1 \%$ ). 
Em relação aos escores dos oito domínios do questionário SF-36, o aspecto social mostrou o maior valor $(76,8 \pm 25,6)$ enquanto $o$ estado geral de saúde apresentou o valor mais baixo $(57,2 \pm 18,3)$.

A Tabela 1 apresenta a caracterização dos dados sociodemográficos dos indivíduos.

Tabela1- Caracterização dos dados sociodemográficos da amostra.

\begin{tabular}{lc}
\hline Variáveis & $\mathbf{n}=\mathbf{1 8 5}$ \\
\hline Idade (anos) - média \pm DP & $70,5 \pm 7,1$ \\
Faixa etária - n (\%) & \\
60 a 69 anos & $93(50,3)$ \\
70 a 79 anos & $70(37,8)$ \\
80 anos ou mais & $22(11,9)$ \\
Sexo - n (\%) & \\
Masculino & $10(5,4)$ \\
Feminino & $175(94,6)$ \\
Cor - n (\%) & \\
Branca & $91(49,2)$ \\
Negra & $69(37,3)$ \\
Parda & $25(13,5)$ \\
Nível de escolaridade - n (\%) & \\
Analfabeto & $21(11,4)$ \\
Fundamental incompleto & $102(55,1)$ \\
Fundamental completo/Médio incompleto & $28(15,1)$ \\
Médio completo ou mais & $34(18,4)$ \\
Situação Conjugal - $\mathrm{n}$ (\%) & \\
Casado & $40(21,6)$ \\
Divorciada & $19(10,3)$ \\
Solteiro & $48(25,9)$ \\
Viúvo & $78(42,2)$ \\
IMC (kg/m²) - média \pm DP & $29,7 \pm 5,9$ \\
Classificação do IMC - n (\%) & \\
Baixo peso & $22(11,9)$ \\
Eutrofia & $51(27,6)$ \\
Sobrepeso & $36(19,5)$ \\
Obesidade & $76(41,1)$ \\
Escore SF-36 - média \pm DP & \\
Capacidade Funcional & $64,6 \pm 26,3$ \\
Limitação por aspectos físicos & $64,7 \pm 39,9$ \\
Dor & $58,8 \pm 28,0$ \\
Estado Geral de Saúde & $57,2 \pm 18,3$ \\
Vitalidade & $66,2 \pm 23,7$ \\
Aspectos Sociais & $76,8 \pm 25,6$ \\
Limitação por Aspectos Emocionais & $66,5 \pm 41,5$ \\
\hline
\end{tabular}

Fonte: Elaborado pela autora 
Analisando a associação dos escores de QV relacionados aos oito domínios do questionário SF-36, os indivíduos obesos apresentaram pior escore de QV nos domínios capacidade funcional e limitação por aspectos físicos, quando comparados aos idosos com baixo peso, eutrofia e sobrepeso ( $p<0,001$ $e p=0,042$ ), respectivamente).

A Tabela 2 apresenta a associação dos escores de QV com o estado nutricional dos indivíduos e a Figura 1 caracteriza a avaliação do escore SF-36, relacionado ao domínio capacidade funcional, conforme a classificação do IMC $(p<0,001)$.

Tabela 2- Associação dos escores de qualidade de vida com estado nutricional.

\begin{tabular}{lccccc}
\hline Escore SF-36 & $\begin{array}{c}\text { Baixo peso } \\
(\mathbf{n}=\mathbf{2 2})\end{array}$ & $\begin{array}{c}\text { Eutrofia } \\
(\mathbf{n}=\mathbf{5 1})\end{array}$ & $\begin{array}{c}\text { Sobrepeso } \\
(\mathbf{n}=\mathbf{3 6})\end{array}$ & $\begin{array}{c}\text { Obesidade } \\
(\mathbf{n = 7 6 )}\end{array}$ & $\boldsymbol{p}$ \\
\cline { 2 - 5 } & Média $\pm \mathrm{DP}$ & Média $\pm \mathrm{DP}$ & Média $\pm \mathrm{DP}$ & Média $\pm \mathrm{DP}$ & \\
\hline Capacidade Funcional & $69,8 \pm 28,3^{\mathrm{b}}$ & $73,3 \pm 23,7^{\mathrm{b}}$ & $69,0 \pm 25,9^{\mathrm{b}}$ & $55,1 \pm 25,0^{\mathrm{a}}$ & $\mathbf{< 0 , 0 0 1}$ \\
Limitação por Aspectos & $62,5 \pm 44,2^{\mathrm{ab}}$ & $70,1 \pm 39,4^{\mathrm{ab}}$ & $77,1 \pm 35,0^{\mathrm{b}}$ & $55,9 \pm 39,9^{\mathrm{a}}$ & $\mathbf{0 , 0 4 2}$ \\
Físicos & & & & & \\
Dor & $66,5 \pm 30,1$ & $60,3 \pm 25,9$ & $62,9 \pm 27,6$ & $53,5 \pm 28,5$ & 0,148 \\
Estado Geral de Saúde & $61,2 \pm 17,4$ & $58,1 \pm 18,3$ & $58,1 \pm 18,2$ & $55,1 \pm 18,6$ & 0,519 \\
Vitalidade & $66,1 \pm 22,9$ & $68,1 \pm 20,3$ & $71,3 \pm 23,3$ & $62,6 \pm 26,0$ & 0,290 \\
Aspectos Sociais & $77,8 \pm 22,1$ & $74,3 \pm 26,3$ & $84,0 \pm 23,1$ & $74,8 \pm 26,9$ & 0,278 \\
Limitação por Aspectos & $68,2 \pm 45,4$ & $67,3 \pm 39,2$ & $72,2 \pm 38,6$ & $62,7 \pm 43,5$ & 0,715 \\
Emocionais & & & & & \\
Saúde Mental & $67,1 \pm 21,3$ & $68,5 \pm 22,8$ & $75,3 \pm 19,7$ & $69,1 \pm 25,3$ & 0,463 \\
\hline
\end{tabular}

a,b Letras iguais não diferem pelo teste de Student Newman-Keuls a 5\% de significância Fonte: Elaborado pelos autores

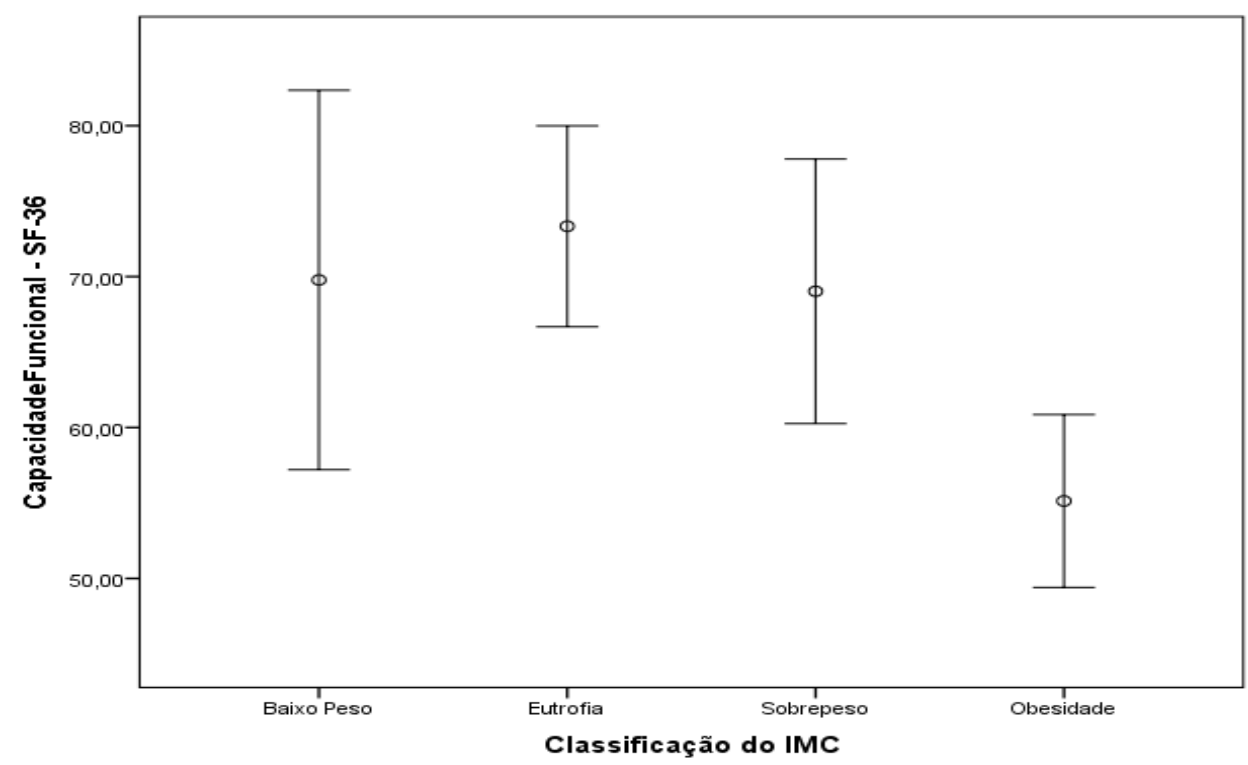

Figura 1. Avaliação do escore SF-36, relacionado ao domínio capacidade funcional, conforme a classificação do IMC $(p<0,001)$. 


\section{DISCUSSÃO}

Este estudo, cujo objetivo foi avaliar a associação entre o estado nutricional e a QV da população idosa atendida pelo Projeto Passos da Longevidade do Banco de Alimentos do RS demonstrou que a capacidade funcional e a limitação por aspectos físicos mostraram uma associação significativa ( $p<0,001$ e $p=0,042$, respectivamente) com obesidade, sinalizando que os indivíduos obesos apresentaram pior capacidade funcional quando comparados com aqueles com sobrepeso, eutrofia e baixo peso e maior limitação por aspectos físicos em relação aos idosos com sobrepeso.

Neste estudo, a prevalência de indivíduos do sexo feminino foi alta (94,6\%). Um estudo com 113 idosos cadastrados no Grupo de Convivência do Serviço Social do Comércio na Paraíba avaliou a QV entre os indivíduos trabalhadores e aposentados e a prevalência de mulheres foi de $62 \%$ e $87 \%$, respectivamente (COSTA et al., 2018). Um trabalho austríaco conduzido por Luger et al. (2016), investigaram a QV de 83 idosos atendidos em seus próprios domicílios e encontraram $86 \%$ de mulheres na amostra (LUGER et al., 2016). $O$ predomínio de mulheres no nosso estudo e em outros semelhantes pode estar associado a maior atenção com a saúde e o autocuidado, levando as mulheres a procurar mais regularmente grupos de convivência e atendimentos de saúde quando comparadas aos homens (LIMA et al., 2017).

A baixa escolaridade, caracterizada pelo ensino fundamental incompleto, presente em $55,1 \%$ dos idosos deste estudo, corrobora com os dados encontrados em outros trabalhos, assim como no estudo de Pereira et al., no qual os autores encontraram uma prevalência de $51,9 \%$ dos indivíduos com o ensino fundamental incompleto (PEREIRA et al., 2015). No estudo de Costa et al., também foram encontradas prevalências de $48 \%$ e $61,9 \%$ de idosos com ensino fundamental incompleto, entre aqueles com atividade laboral e os aposentados, respectivamente (COSTA et al., 2018). A prevalência de idosos com baixa escolaridade pode ser justificado pelas suas condições de vida, pois permanecem poucos anos na escola para ajudar a trazer proventos para a família, se abdicando da escola para trabalhar na infância (ALCÂNTARA et al., 2016). 
No presente estudo, não foi verificada associação entre estado nutricional e as variáveis faixa etária, sexo, cor, nível de escolaridade e situação conjugal.

Um dado importante na amostra estudada é o número elevado de idosos com excesso de peso (60,6\%); 36 (19,5\%) indivíduos apresentaram sobrepeso e $76(41,1 \%)$ obesidade. O estudo de Freitas et al, com 186 idosos integrantes de um grupo de convivência da terceira idade no município de Estrela (RS) encontrou uma prevalência de excesso de peso de 53,2\% (18,3\% com sobrepeso e 34,9\% com obesidade) (FREITAS et al., 2017.) Outro estudo avaliou 49 idosos de ambos os sexos, participantes de um grupo de convivência em Minas Gerais e os autores também observaram uma predominância de excesso de peso (46,9\% de sobrepeso e 18,3\% obesos) (FREITAS et al., 2019).

Analisando a associação dos escores de QV relacionados aos oito domínios do questionário SF-36, em geral, os indivíduos obesos apresentaram pior QV em praticamente todos os domínios, com exceção dos aspectos sociais e de saúde mental. Souza et al., avaliaram a QV de 27 idosos com sobrepeso e obesidade, acompanhados em uma Unidade de Saúde no Ceará pelo questionário WHOQOL-OLD. Os resultados mostraram baixo escore no aspecto participação social, resultado este diferente do nosso estudo que sinalizou que os idosos com sobrepeso tiveram maior escore no domínio aspectos sociais (SOUZA et al., 2018).

O estudo conduzido por Tavares et al., com 370 idosos teve como objetivo verificar a associação do excesso de peso com a capacidade funcional, morbidades autorreferidas e QV de idosos residentes em área rural, através de diferentes instrumentos (Escalas Katz e Lawton; World Health Organization Quality of Life - BREF e World Health Organization Quality of Life Assessment for Older Adults). Os resultados demostraram que o excesso de peso foi associado negativamente com a capacidade funcional, mas positivamente com o domínio relações sociais (TAVARES et al., 2018). Resultados semelhantes foram encontrados em um trabalho chinês que teve o objetivo de estudar a associação entre o IMC e a QV, mensurada através do SF-36, entre 21.218 adultos com 18 anos ou mais, sendo 1.780 indivíduos com idades entre 60 e 90 anos. No geral, os resultados mostraram que os escores médios de QV aumentaram com o aumento do IMC. Comparado ao grupo de indivíduos eutróficos, aqueles com obesidade grau I tiveram pontuações significativamente 
mais altas nos domínios saúde mental, aspectos físicos, saúde geral, vitalidade e aspectos sociais (Zhu et al., 2015).

O trabalho de Acar Tek e Karaçil-Ermumcu (2018), realizado com 407 idosos na Turquia, teve como objetivo examinar fatores como anorexia e estado nutricional e a sua relação com a QV utilizando o questionário SF-36. Foi observado que, quanto maior o IMC menores foram os escores de QV. No nosso trabalho, os indivíduos obesos apresentaram piores escores de QV em todos os domínios com exceção dos aspectos sociais e de saúde mental (ACAR TEK e KARACI-ERMUMCU, 2018).

You et al. (2018), em um trabalho chinês com 10.527 idosos teve como objetivo avaliar a relação entre o IMC e a QV utilizando o instrumento EQ-5D-3L. Os resultados do estudo sinalizaram que o baixo peso foi um fator de risco para a pior QV em ambos os sexos, enquanto o excesso de peso impactou positivamente na QV dos homens e negativamente na das mulheres (YOU et al., 2018).

A relação entre o IMC e a QV foi estudada em um grupo de 3981 homens e 3099 mulheres, com 65 anos ou mais, em um projeto da Chicago Heart Association, atavés do Questonário Status de Saúde-12. Os resultados mostraram que após o ajuste das variáveis para idade, raça, educação, tabagismo e ingestão de álcool, a obesidade foi associada a menor percepção de saúde e pior funcionamento físico e social (apenas nas mulheres), mas não prejudicou a saúde mental. $O$ excesso de peso foi associado ao comprometimento do bem-estar físico apenas entre as mulheres. Tanto os homens como as mulheres com baixo peso relataram comprometimento do bemestar físico, social e mental (YAN et al., 2004).

Neste estudo, a capacidade funcional e a limitação por aspectos físicos tiveram associação positiva com obesidade, sinalizando que os indivíduos obesos apresentaram pior capacidade funcional quando comparados com aqueles com sobrepeso, eutrofia e baixo peso e maior limitação por aspectos físicos em relação aos idosos com sobrepeso.

O estudo conduzido por Garcia et al., com indivíduos cuja média de idade foi 61 anos e que utilizou o questionário SF-12 como instrumento para avaliar a QV, demonstrou que o valor do IMC foi inversamente associado com os escores dos domínios físico e mental, ou seja, quanto maior o IMC, menores os scores, 
e consequentemente, pior a QV, corroborando com os resultados relacionados aos aspectos físicos encontrados no presente estudo (GARCIA et al., 2016).

Outro trabalho, realizado com 156 idosos de um grupo de terceira idade de um município do interior do RS, utilizou o questionário WOQOL-BREF para avaliar a QV. Este instrumento apresenta 26 perguntas distribuídas em 4 domínios: físico, psicológico, de meio ambiente e de relação social. Os resultados mostraram que o IMC apresentou uma relação inversa com o domínio físico (inclui questões sobre dor, desconforto, energia, fadiga, sono, repouso, mobilidade, atividades de vida diária, dependência de medicação ou tratamentos e capacidade para o trabalho), ou seja, quanto maior o valor do IMC, menor foi o escore para o domínio físico (ADAMI et al., 2015). Estes resultados reforçam os achados do nosso estudo uma vez que a capacidade funcional e a limitação por aspectos físicos também mostraram uma associação significativa com obesidade, indicando que os indivíduos obesos apresentaram pior capacidade funcional quando comparados com aqueles com sobrepeso, eutrofia e baixo peso e maior limitação por aspectos físicos em relação àqueles com sobrepeso.

Abdollahi et al. (2016) em um estudo com 421 idosos teve como objetivo investigar a associação entre obesidade e QV utilizando o questionário SF-36. Os resultados encontrados foram semelhantes ao estudo atual, no qual os indivíduos com excesso de peso apresentaram menores escores em todos os domínios, com exceção de Saúde Mental, quando comparados aos indivíduos eutróficos (ABDOLLAHI et al., 2016).

O estudo realizado por Silva et al., avaliou a relação do risco nutricional com a capacidade funcional, através da Avaliação das Atividades Básicas e Instrumentais de Vida Diária (AABIVD), mensuradas pelo Índice de Katz e pela Escala de Lawton-Brody em 27 idosos matriculados na Universidade da Maturidade no Tocantins. Foi observado uma relação inversa entre estas variáveis, ou seja, os idosos com menores pontuações na Mini Avaliação Nutricional (MAN) apresentaram capacidade funcional mais baixa e maior fragilidade, o que não corrobora com os resultados do nosso estudo onde os menores escores na capacidade funcional foram observados nos indivíduos obesos seguido daqueles com baixo peso (SILVA et al., 2019).

Lee et al., estudaram 542 idosos selecionados em Centros de Saúde e Hospitais Universitários na Coreia do Sul e avaliaram a associação entre IMC e 
QV mensurada pelo questionário SF-36. Os resultados sinalizaram que não houve associação entre maiores valores de IMC com pior QV, resultados também diferentes dos achados do presente estudo (LEE et al., 2017). Em contrapartida, o estudo de Souza et al., avaliou o estado nutricional, as condições de saúde e as suas influências na capacidade funcional de 47 idosos participantes de um centro de convivência no Mato Grosso do Sul, através da AABIVD, mensuradas pelo Índice de Katz e pela Escala de Lawton-Brody. Foi observado que valores maiores de IMC foram correlacionados negativamente com a capacidade funcional, corroborando com os resultados encontrados no presente estudo (SOUZA et al., 2018).

Algumas limitações deste estudo devem ser destacadas. Em primeiro lugar, a população estudada, por ter sido selecionada a partir de grupos de convivência de idosos residentes na periferia da cidade, pode não ser representativa do universo de idosos. O SF-36, embora seja um instrumento bem desenhado, com boa reprodutibilidade, validade e suscetibilidade a alterações, suas questões são voltadas para os aspectos físicos e outros que ocorrem como consequência de doenças, sendo, portanto, mais específico para grupos populacionais com determinadas patologias, embora a maioria dos idosos apresente doenças, especialmente crônico-degenerativas. Considerando-se que a QV é influenciada por diferentes fatores, a delimitação de apenas aspectos físicos e relativos a doenças pode não refletir de forma abrangente a percepção de QV desses indivíduos.

\section{CONCLUSÃO}

Neste estudo, os indivíduos obesos apresentaram pior escore de QV nos domínios capacidade funcional e limitação por aspectos físicos, quando comparados aos idosos com baixo peso, eutrofia e sobrepeso.

\section{CONFLITO DE INTERESSES}

Não existem conflitos de interesse 


\section{REFERÊNCIAS}

ABDOLLAHI, S. et al. The association between obesity and quality of life among the elderly. International Journal of Health Studies, v. 2, p. 17-22, 2016.

ACAR, T.E.K; KARACI-ERMUMCU, M.S. Determinants of health related quality of life in home dwelling elderly population: appetite and nutritional status. J Nutr Health Aging, v. 22, p. 996-1002, 2018.

ADAMI, F.S; FEIL, C.C; BOSCO, S.M.D. Estado nutricional relacionado à qualidade de vida em idosos. Rev Soc Bras Clin Med, v. 12, p. 28-40, 2015.

ALCÂNTARA, A.O; CAMARANO, A.A; GIACOMINI, C.K. Política nacional do idoso: velhas e novas questões. Rio de Janeiro, Instituto de Pesquisa Econômica Aplicada 2016; 63-100p.

ANDRADE, F.L.J.P. et al. Incapacidade cognitiva e fator H.es associados em idosos institucionalizados em Natal, RN, Brasil. Rev Bras Geriatr Geronto, v. 20, p.186-197, 2017.

COSTA, I.P. et al. Qualidade de vida de idosos e sua relação com o trabalho. Rev Gaúcha Enferm, vol.39, p.1-9, 2018.

FREITAS, A.P. et al. Relação da qualidade de vida com o estado nutricional de idosos. Rev Bras Qual Vida, v.9, p.30-44, 2017.

FREITAS, R.F. et al. Índice de massa corporal e os fatores associados em idosos participantes de grupo de convivência. Rev Univap; v. 25, p. 110-121, 2019.

GARCIA, C.A.M.S; MORETTO, M.C; GUARIENTO, M.E. Estado nutricional e qualidade de vida em idosos. Rev Soc Bras Clin Med, v. 14, p. 52-60, 2016.

HOLZ, A.W. et al. Prevalência de déficit cognitivo e fatores associados entre idosos de Bagé, Rio Grande do Sul, Brasil. Rev Bras Epidemiol, v.16, p. 880888, 2013.

KEHOE, L; WALTON, J; FLYNN, A. Nutritional challenges for older adults in Europe: current status and future directions. Proceedings of the Nutrition Society, v.78, p. 221-233, 2018.

LEE, G. et al. Obesity and Quality of Life in the Elderly. Korean J Fam Med, v. 38, p. 181-191, 2017.

LIMA, A.P.M. et al. Avaliação nutricional de idosos residentes em instituições de longa permanência. Rev Baiana Enferm, v. 31, p. 1-9, 2017. 
LUGER, E. et al. Association between nutritional status and quality of life in (pre) frail community-dwelling older persons. The Journal of Frailty \& Aging, v. 5, p. 141-148, 2016.

MAHAN, K; ESCOTT-STUP, S; RAYMOND, J.K. Alimentos, Alimentação e Dietoterapia. Rio de Janeiro, Editora: Saunders, 13 ed. 2013.

Organización Panamericana de la Salud. División de Promoción y Protección de la Salud (HPP). Encuesta Multicentrica salud beinestar y envejecimiento (SABE) em América Latina el Caribe: Informe Preliminar. In: XXXVI Reunión del Comité Asesor de Investigaciones em Salud; 9-11 jun 2001; Kingston, Jamaica: OPAS, 2002. Disponível em: www.opas.org/program/sabe.htm.

PAULI, T.P. et al. Saúde bucal de idosos com 80 anos ou mais: condição, autopercepção e utilização de serviços odontológicos. Rev Odontol UNESP, v.47, p. 291-297, 2018.

PEREIRA, D.S; NOGUEIRA, J.A.D; SILVA, C.A.B. Qualidade de vida e situação de saúde de idosos: um estudo de base populacional no Sertão Central do Ceará. Rev Bras Geriatr Geronto, v.8, p. 893-908, 2015.

SILVA, L.O. et al. Relação do Estado Nutricional com capacidade funcional e fragilidade em idosos. Revista Humanidades e Inovação, v.6, p. 138-149, 2019.

SILVA, S.M.C.S; MURA, J.D.P. Tratado de Alimentação, Nutrição e Dietoterapia. São Paulo, Editora: Payá, 2 ed. 2011.

SOUZA, L.B. et al. Excesso de peso influencia negativamente a capacidade funcional de idosos em atividades cotidianas. Geriatr Gerontol Aging, v. 13, p. $62-68,2018$.

SOUZA Y.P. et al. A qualidade de vida de idosos com obesidade ou sobrepeso. Rev Bras Cie Saúde, v. 22, p. 155-164, 2018.

TAFT, C; KARLSSON, J; SULLIVAN, M. SF-36 summary components scores accurately summarize subscale scores? Quality of Life Research; v.10, p. 395-404, 2001.

TAVARES, D.M.S. et al. Excesso de peso em idosos rurais: associação com as condições de saúde e qualidade de vida. Ciência \& Saúde Coletiva, v.23, p. 913-922, 2018.

YAN, L.L. et al. BMI and Health-Related Quality of Life in Adults 65 Years and Older. Obes Res, v. 2, p. 69-76, 2004.

YOU, H. et al. Association between body mass index and health-related quality of life among Chinese elderly - evidence from a community-based study. BMC Public Health, v. 18, p. 1-9, 2018. 
ZHU, Y. et al. Association between body mass index and health-related quality of life: The "obesity paradox" in 21,218 adults of the chinese general population. PLoS One; v. 10, 2015: Disponível em: https://doi.org/10.1371/ jornal.pone.0130613. 